Proyecciones Journal of Mathematics

Vol. 34, No 3, pp. 255-276, September 2015.

Universidad Católica del Norte

Antofagasta - Chile

\title{
Asymptotic stability in totally nonlinear neutral difference equations
}

\author{
Abdelouaheb Ardjouni \\ University Souk Ahras, Algeria \\ and \\ Ahcene Djoudi \\ University Annaba, Algeria \\ Received : February 2015. Accepted : July 2015
}

\begin{abstract}
In this paper we use fixed point method to prove asymptotic stability results of the zero solution of the totally nonlinear neutral difference equation with variable delay

$$
\triangle x(n)=-a(n) f(x(n-\tau(n)))+\triangle g(n, x(n-\tau(n))) .
$$

An asymptotic stability theorem with a sufficient condition is proved, which improves and generalizes some results due to Raffoul (2006) [23], Yankson (2009) [27], Jin and Luo (2009) [17] and Chen (2013) $[9]$.
\end{abstract}

Subjclass [2000] : 39A30, 39A70.

Keywords : Fixed point, Stability, Neutral difference equations, Variable delay. 


\section{INTRODUCTION}

Certainly, the Lyapunov direct method has been, for more than 100 years, the efficient tool for the study of stability properties of ordinary, functional, partial differential and difference equations. Nevertheless, the application of this method to problems of stability in differential and difference equations with delay has encountered serious difficulties if the delay is unbounded or if the equation has unbounded terms ([6],[7],[11]-[13],[15],[25]). Recently, Burton, Furumochi, Zhang, Raffoul, Islam, Yankson and others have noticed that some of these difficulties vanish or might be overcome by means of fixed point theory (see [1],[2],[3],[6],[7],[9],[16],[17], [23],[24],[27]-[29]). The fixed point theory does not only solve the problem on stability but has a significant advantage over Lyapunov's direct method. The conditions of the former are often averages but those of the latter are usually pointwise (see [6]). Yet the stability theory of difference equations with/without delay has been considered by many authors without the application of Lyapunov and fixed point methods, see the papers [4],[5],[8],[14],[19]-[22],[30].

In this paper, we consider the nonlinear neutral difference equation with variable delay

$$
\triangle x(n)=-a(n) f(x(n-\tau(n)))+\triangle g(n, x(n-\tau(n))),
$$

with the initial condition

$$
x(n)=\psi(n) \text { for } n \in\left[m\left(n_{0}\right), n_{0}\right] \cap \mathbf{Z},
$$

where $\psi:\left[m\left(n_{0}\right), n_{0}\right] \cap \mathbf{Z} \rightarrow \mathbf{R}$ is a bounded sequence and for $n_{0} \geq 0$,

$$
m\left(n_{0}\right)=\inf \left\{n-\tau(n), n \geq n_{0}\right\} .
$$

Throughout this paper we assume that $a: \mathbf{Z}^{+} \rightarrow \mathbf{R}, f: \mathbf{R} \rightarrow \mathbf{R}$ and $\tau: \mathbf{Z}^{+} \rightarrow \mathbf{Z}^{+}$with $n-\tau(n) \rightarrow \infty$ as $n \rightarrow \infty$. The function $g(n, x)$ is locally Lipschitz continuous in $x$. That is, there is positive constant $E$ so that if $|x| \leq l$ for some positive constant $l$ then

$$
|g(n, x)-g(n, y)| \leq E|x-y| .
$$

We also assume that

$$
g(n, 0)=0 .
$$

Special cases of (1.1) have been considered and investigated by many authors. For example, Raffoul in [23] and Yankson in [27] studied the equation 


$$
\triangle x(n)=-a(n) x(n-\tau(n)),
$$

and proved the following theorems.

Theorem A (Raffoul [23]). Suppose that $\tau(n)=r$ and $a(n+r) \neq 1$ and there exists a constant $\alpha<1$ such that

$\sum_{s=n-r}^{n-1}|a(s+r)|+\sum_{s=0}^{n-1}\left(|a(s+r)|\left|\prod_{k=s+1}^{n-1}[1-a(k+r)]\right| \sum_{u=s-r}^{s-1}|a(u+r)|\right) \leq \alpha$,

for all $n \in \mathbf{Z}^{+}$and $\prod_{s=0}^{n-1}[1-a(s+r)] \rightarrow 0$ as $n \rightarrow \infty$. Then, for every small initial sequence $\psi:[-r, 0] \cap \mathbf{Z} \rightarrow \mathbf{R}$, the solution $x(n)=x(n, 0, \psi)$ of (1.4) is bounded and tends to zero as $n \rightarrow \infty$.

Theorem B (Yankson [27]). Suppose that $Q(n) \neq 0$ for all $n \in\left[n_{0}, \infty\right) \cap \mathbf{Z}$, the inverse sequence $g$ of $n-\tau(n)$ exists and there exists a constant $\alpha \in$ $(0,1)$ for all $n \in\left[n_{0}, \infty\right) \cap \mathbf{Z}$ such that

$\sum_{s=n-\tau(n)}^{n-1}|a(g(s))|+\sum_{s=n_{0}}^{n-1}\left(|1-Q(s)|\left|\prod_{k=s+1}^{n-1} Q(k)\right| \sum_{u=s-\tau(s)}^{s-1}|a(g(u))|\right) \leq \alpha$,

where $Q(n)=1-a(g(n))$. Then the zero solution of (1.4) is asymptotically stable if $\prod_{s=n_{0}}^{n-1} Q(s) \rightarrow 0$ as $n \rightarrow \infty$.

Obviously, Theorem B improves and generalizes Theorem A. On other hand, Jin and Luo in [17] and Chen in [9] considered the generalized form of (1.4),

$$
\triangle x(n)=-a(n) f(x(n-\tau(n))),
$$

and obtained the following theorems.

Theorem C (Jin and Luo [17]). Suppose that $\tau(n)=r$. Let $f$ be odd, increasing on $[0, l]$, satisfy a Lipschitz condition, and let $x-f(x)$ be nondecreasing on $[0, l]$. Suppose that $|a(n)|<1$ and for each $l_{1} \in(0, l]$ we have 


$$
\begin{aligned}
& \left|l_{1}-f\left(l_{1}\right)\right| \sup _{n \in \mathbf{Z}^{+}} \sum_{s=0}^{n-1}|a(s+r)| \prod_{k=s+1}^{n-1}[1-a(k+r)] \\
+ & f\left(l_{1}\right) \sup _{n \in \mathbf{Z}^{+}} \sum_{s=0}^{n-1}|a(s+r)| \prod_{k=s+1}^{n-1}[1-a(k+r)] \sum_{u=s-r}^{n-1}|a(u+r)| \\
+ & f\left(l_{1}\right) \sup _{n \in \mathbf{Z}^{+}} \sum_{s=n-r}^{n-1}|a(s+r)| \leq \alpha l_{1} .
\end{aligned}
$$

Then the zero solution of (1.7) is stable.

Theorem D (Chen [9]). Suppose that the following conditions are satisfied

(i) the function $f$ is odd, increasing on $[0, l]$,

(ii) $f(x)$ and $x-f(x)$ satisfy a Lipschitz condition with constant $K$ on an interval $[-l, l]$, and $x-f(x)$ is nondecreasing on $[0, l]$,

(iii) the inverse function $g(n)$ of $n-\tau(n)$ exists and $|a(g(n))|<1$,

(iv) there exists a constant $\alpha \in(0,1)$ for all $n \in \mathbf{Z}^{+}$such that

$$
\begin{aligned}
& \sum_{s=0}^{n-1}|a(g(s))| \prod_{k=s+1}^{n-1}[1-a(g(k))]+\sum_{s=n-r}^{n-1}|a(g(s))| \\
& +\sum_{s=0}^{n-1}|a(g(s))| \prod_{k=s+1}^{n-1}[1-a(g(k))] \sum_{u=s-r}^{n-1}|a(g(u))| \\
& \quad \leq \alpha .
\end{aligned}
$$

Then the zero solution of (1.7) is asymptotically stable if $\prod_{k=0}^{n-1}[1-a(g(k))] \rightarrow 0$ as $n \rightarrow \infty$.

Obviously, Theorem D improves Theorem C.

Our purpose here is to improve Theorems A-D and extend it to investigate a wide class of nonlinear neutral difference equation with variable delay presented in (1.1). Our results are obtained with no need of further assumptions on the inverse of sequence $n-\tau(n)$, so that for a given bounded initial sequence $\psi$ a mapping $P$ for (1.1) is constructed in such a way to map a, carefully chosen, complete metric space $S_{\psi}^{\epsilon}$ into itself on which $P$ is a contraction mapping possessing a fixed point. This procedure will enable us to establish and prove by means of the contraction mapping theorem an asymptotic stability theorem for the zero solution of (1.1) with a less restrictive conditions. It is important to note that, in our consideration, the neutral term $\triangle g(n, x(n-\tau(n)))$ of (1.1) produces nonlinearity in the 
neutral term $\triangle x(n-\tau(n))$. While, the neutral term in [3] enters linearly. As a consequence, we have performed an appropriate analysis which is different from that used in [3] to construct the mapping in order to employ fixed point theorems. For details on contraction mapping principle we refer the reader to [26] and for more on the calculus of difference equations, we refer the reader to [10] and [18]. The results presented in this paper improve and generalize the main results in [9],[17],[23],[27].

\section{Main results}

For a fixed $n_{0}$, we denote $D\left(n_{0}\right)$ the set of bounded squences $\psi:\left[m\left(n_{0}\right), n_{0}\right] \cap$ $\mathbf{Z} \rightarrow \mathbf{R}$ with the norm $|\psi|_{0}=\max \left\{|\psi(n)|: n \in\left[m\left(n_{0}\right), n_{0}\right] \cap \mathbf{Z}\right\}$. For each $\left(n_{0}, \psi\right) \in \mathbf{Z}^{+} \times D\left(n_{0}\right)$, a solution of (1.1) through $\left(n_{0}, \psi\right)$ is a sequence $x$ : $\left[m\left(n_{0}\right), \infty\right) \cap \mathbf{Z} \rightarrow \mathbf{R}$ such that $x$ satisfies $(1.1)$ on $\left[n_{0}, \infty\right) \cap \mathbf{Z}$ and $x=\psi$ on $\left[m\left(n_{0}\right), n_{0}\right] \cap \mathbf{Z}$. We denote such a solution by $x(n)=x\left(n, n_{0}, \psi\right)$. For each $\left(n_{0}, \psi\right) \in \mathbf{Z}^{+} \times D\left(n_{0}\right)$, there exists a unique solution $x(n)=x\left(n, n_{0}, \psi\right)$ of (1.1) defined on $\left[m\left(n_{0}\right), \infty\right) \cap \mathbf{Z}$.

Let $h:\left[m\left(n_{0}\right), \infty\right) \cap \mathbf{Z} \rightarrow \mathbf{R}$ be an arbitrary sequence. Rewrite (1.1) as $\triangle x(n)=-h(n) f(x(n))+\triangle_{n} \sum_{s=n-\tau(n)}^{n-1} h(s) f(x(s))$

$$
\begin{aligned}
&+\{h(n-\tau(n))-a(n)\} f(x(n-\tau(n)))+\triangle g(n, x(n-\tau(n))) \\
&=-h(n) x(n)+h(n)[x(n)-f(x(n))]+\triangle_{n} \sum_{s=n-\tau(n)}^{n-1} h(s) f(x(s)) \\
&+\{h(n-\tau(n))-a(n)\} f(x(n-\tau(n)))+\triangle g(n, x(n-\tau(n))),
\end{aligned}
$$

where $\triangle_{n}$ represents that the difference is with respect to $n$. If we let $H(n)=1-h(n)$ then $(2.1)$ is equivalent to

$$
\begin{gathered}
x(n+1)=H(n) x(n)+h(n)[x(n)-f(x(n))]+\triangle_{n} \sum_{s=n-\tau(n)}^{n-1} h(s) f(x(s)) \\
\quad+\{h(n-\tau(n))-a(n)\} f(x(n-\tau(n)))+\triangle g(n, x(n-\tau(n))) .
\end{gathered}
$$


In the process, for any sequence $x$, we denote

$$
\sum_{k=a}^{b} x(k)=0 \text { and } \prod_{k=a}^{b} x(k)=1 \text { for any } a>b .
$$

Lemma 2.1. Suppose that $H(n) \neq 0$ for all $n \in\left[n_{0}, \infty\right) \cap \mathbf{Z}$. Then $x$ is a solution of equation (1.1) if and only if

$$
\begin{aligned}
& \mathrm{x}(n)=\left\{x\left(n_{0}\right)-g\left(n_{0}, x\left(n_{0}-\tau\left(n_{0}\right)\right)\right)-\sum_{s=n_{0}-\tau\left(n_{0}\right)}^{n_{0}-1} h(s) f(x(s))\right\} \\
\times & \prod_{u=n_{0}}^{n-1} H(u) \\
+ & \sum_{s=n_{0}}^{n-1} h(s) \prod_{u=s+1}^{n-1} H(u)[x(s)-f(x(s))]+\sum_{s=n-\tau(n)}^{n-1} h(s) f(x(s)) \\
- & \sum_{s=n_{0}}^{n-1}\{h(s)\} \prod_{u=s+1}^{n-1} H(u) \sum_{v=s-\tau(s)}^{s-1} h(v) f(x(v)) \\
+ & \sum_{s=n_{0}}^{n-1} \prod_{u=s+1}^{n-1} H(u)\{h(s-\tau(s))-a(s)\} f(x(s-\tau(s))) \\
+ & g(n, x(n-\tau(n)))-\sum_{s=n_{0}}^{n-1}\{h(s)\} \prod_{u=s+1}^{n-1} H(u) g(s, x(s-\tau(s))) .
\end{aligned}
$$

Proof. Let $x$ be a solution of (1.1). By multiplying both sides of (2.2) by $\prod_{u=n_{0}}^{n}[H(u)]^{-1}$ and by summing from $n_{0}$ to $n-1$ we obtain

$$
\begin{aligned}
& \sum_{s=n_{0}}^{n-1} \triangle\left[\prod_{u=n_{0}}^{s-1}[H(u)]^{-1} x(s)\right] \\
& =\sum_{s=n_{0}}^{n-1} \prod_{u=n_{0}}^{s}[H(u)]^{-1} h(s)[x(s)-f(x(s))] \\
& +\sum_{s=n_{0}}^{n-1} \prod_{u=n_{0}}^{s}[H(u)]^{-1} \triangle_{s} \sum_{v=s-\tau(s)}^{s-1} h(v) f(x(v))
\end{aligned}
$$




$$
\begin{aligned}
& +\sum_{s=n_{0}}^{n-1} \prod_{u=n_{0}}^{s}[H(u)]^{-1}\{h(s-\tau(s))-a(s)\} f(x(s-\tau(s))) \\
& +\sum_{s=n_{0}}^{n-1} \prod_{u=n_{0}}^{s}[H(u)]^{-1} \triangle g(s, x(s-\tau(s))) .
\end{aligned}
$$

As a consequence, we arrive at

$$
\begin{aligned}
& \prod_{u=n_{0}}^{n-1}[H(u)]^{-1} x(n)-\prod_{u=n_{0}}^{n_{0}-1}[H(u)]^{-1} x\left(n_{0}\right) \\
= & \sum_{s=n_{0}}^{n-1} \prod_{u=n_{0}}^{s}[H(u)]^{-1} h(s)[x(s)-f(x(s))] \\
+ & \sum_{s=n_{0}}^{n-1} \prod_{u=n_{0}}^{s}[H(u)]^{-1} \triangle_{s} \sum_{v=s-\tau(s)}^{s-1} h_{j}(v) f_{j}(x(v)) \\
+ & \sum_{s=n_{0}}^{n-1} \prod_{u=n_{0}}^{s}[H(u)]^{-1}\{h(s-\tau(s))-a(s)\} f(x(s-\tau(s))) \\
+ & \sum_{s=n_{0}}^{n-1} \prod_{u=n_{0}}^{s}[H(u)]^{-1} \triangle g(s, x(s-\tau(s))) .
\end{aligned}
$$

By dividing both sides of the above expression by $\prod_{u=n_{0}}^{n-1}[H(u)]^{-1}$ we get

$$
\begin{aligned}
& \mathrm{x}(n)=x\left(n_{0}\right) \prod_{u=n_{0}}^{n-1} H(u) \\
+ & \sum_{s=n_{0}}^{n-1} \prod_{u=s+1}^{n-1} H(u) h(s)[x(s)-f(x(s))] \\
+ & \sum_{s=n_{0}}^{n-1} \prod_{u=s+1}^{n-1} H(u) \triangle_{s} \sum_{v=s-\tau(s)}^{s-1} h(v) f(x(v)) \\
+ & \sum_{s=n_{0}}^{n-1} \prod_{u=s+1}^{n-1} H(u)\{h(s-\tau(s))-a(s)\} f(x(s-\tau(s))) \\
+ & \sum_{s=n_{0}}^{n-1} \prod_{u=s+1}^{n-1} H(u) \triangle g(s, x(s-\tau(s))) .
\end{aligned}
$$

By performing a summation by parts, we have 


$$
\begin{aligned}
& \sum_{s=n_{0}}^{n-1} \prod_{u=s+1}^{n-1} H(u) \triangle_{s} \sum_{v=s-\tau(s)}^{s-1} h(v) f(x(v)) \\
= & \sum_{s=n-\tau(n)}^{n-1} h(s) f(x(s))-\prod_{u=n_{0}}^{n-1} H(u) \sum_{s=n_{0}-\tau\left(n_{0}\right)}^{n_{0}-1} h(s) f(x(s)) \\
- & \sum_{s=n_{0}}^{n-1}\{h(s)\} \prod_{u=s+1}^{n-1} H(u) \sum_{v=s-\tau(s)}^{s-1} h(v) f(x(v)),
\end{aligned}
$$

and

$$
\begin{gathered}
\sum_{s=n_{0}}^{n-1} \prod_{u=s+1}^{n-1} H(u) \triangle g(s, x(s-\tau(s))) \\
=-g\left(n_{0}, x\left(n_{0}-\tau\left(n_{0}\right)\right)\right) \prod_{u=n_{0}}^{n-1} H(u)+g(n, x(n-\tau(n))) \\
-\sum_{s=n_{0}}^{n-1}\{h(s)\} \prod_{u=s+1}^{n-1} H(u) g(s, x(s-\tau(s))) .
\end{gathered}
$$

Finally, substituting (2.5) and (2.6) into (2.4) completes the proof.

From equation (2.3) we shall derive a fixed point mapping $P$ for (1.1). But the challenge here is to choose a suitable metric space of sequences on which the map $P$ can be defined. Below a weighted metric on a specific space is defined. Let $C$ be the Banach space of real bounded sequences $\varphi:\left[m\left(n_{0}\right), \infty\right) \cap \mathbf{Z} \rightarrow \mathbf{R}$ with the supremum norm $\|\cdot\|$, that is, for $\varphi \in C$,

$$
\|\varphi\|=\sup \left\{|\varphi(n)|: n \in\left[m\left(n_{0}\right), \infty\right) \cap \mathbf{Z}\right\} .
$$

In other words, we carry out investigations in the complete metric space $(C, d)$ where $d$ denotes the supremum metric $d\left(\varphi_{1}, \varphi_{2}\right)=\left\|\varphi_{1}-\varphi_{2}\right\|$ for $\varphi_{1}, \varphi_{2} \in C$. For a given initial sequence $\psi:\left[m\left(n_{0}\right), n_{0}\right] \cap \mathbf{Z} \rightarrow[-l, l]$ with $l>0$, define the set

$$
S_{\psi}^{l}=\left\{\varphi \in C, \varphi(n)=\psi(n) \text { for } n \in\left[m\left(n_{0}\right), n_{0}\right] \cap \mathbf{Z},|\varphi(n)| \leq l\right\} .
$$

Since $S_{\psi}^{l}$ is a closed subset of $C$, the metric space $\left(S_{\psi}^{l}, d\right)$ is complete. 
Definition 2.2. The zero solution of (1.1) is Lyapunov stable if for any $\epsilon>0$ and any integer $n_{0} \geq 0$ there exists a $\delta>0$ such that $|\psi(n)| \leq \delta$ for $n \in\left[m\left(n_{0}\right), n_{0}\right] \cap \mathbf{Z}$ implies $\left|x\left(n, n_{0}, \psi\right)\right| \leq \epsilon$ for $n \in\left[n_{0}, \infty\right) \cap \mathbf{Z}$.

Theorem 2.3. Define a mapping $P$ on $S_{\psi}^{l}$ as follows, for $\varphi \in S_{\psi}^{l}(P \varphi)(n)=$ $\psi(n)$ if $n \in\left[m\left(n_{0}\right), n_{0}\right] \cap \mathbf{Z}$, while, for $n \in\left[n_{0}, \infty\right) \cap \mathbf{Z}$

$$
\begin{aligned}
(P \varphi) & (n)=\left\{\psi\left(n_{0}\right)-g\left(n_{0}, \psi\left(n_{0}-\tau\left(n_{0}\right)\right)\right)-\sum_{s=n_{0}-\tau\left(n_{0}\right)}^{n_{0}-1} h(s) f(\psi(s))\right\} \\
& \times \prod_{u=n_{0}}^{n-1} H(u) \\
& +\sum_{s=n_{0}}^{n-1} h(s) \prod_{u=s+1}^{n-1} H(u)[\varphi(s)-f(\varphi(s))]+\sum_{s=n-\tau(n)}^{n-1} h(s) f(\varphi(s)) \\
& -\sum_{s=n_{0}}^{n-1} h(s) \prod_{u=s+1}^{n-1} H(u) \sum_{v=s-\tau(s)}^{s-1} h(v) f(\varphi(v)) \\
& +\sum_{s=n_{0}}^{n-1} \prod_{u=s+1}^{n-1} H(u)\{h(s-\tau(s))-a(s)\} f(\varphi(s-\tau(s))) \\
& +g(n, \varphi(n-\tau(n)))-\sum_{s=n_{0}}^{n-1} h(s) \prod_{u=s+1}^{n-1} H(u) g(s, \varphi(s-\tau(s))) .
\end{aligned}
$$

Suppose that (1.2) and (1.3) hold and the following conditions are satisfied,

(i) the function $f$ is odd, increasing on $[0, l]$,

(ii) $f(x)$ and $x-f(x)$ satisfy a Lipschitz condition with constant $K$ on an interval $[-l, l]$, and $x-f(x)$ is nondecreasing on $[0, l]$,

(iii) $|h(n)|<1$ for $n \in\left[m\left(n_{0}\right), \infty\right) \cap \mathbf{Z}$ and $|h(n-\tau(n))-a(n)|<1$, $\rho E<1$ for $n \in\left[n_{0}, \infty\right) \cap \mathbf{Z}, \rho>6$,

(iv) there exist a constant $\alpha \in(0,1)$ for all $n \in\left[n_{0}, \infty\right) \cap \mathbf{Z}$ such that

$f(l)\left\{\sum_{s=n_{0}}^{n-1}|h(s)| \prod_{u=s+1}^{n-1} H(u)+\sum_{s=n-\tau(n)}^{n-1}|h(s)|\right.$

$$
+\sum_{s=n_{0}}^{n-1}|h(s)| \prod_{u=s+1}^{n-1} H(u) \sum_{v=s-\tau(s)}^{s-1}|h(v)|
$$




$$
\begin{aligned}
& \left.+\sum_{s=n_{0}}^{n-1} \prod_{u=s+1}^{n-1} H(u)|h(s-\tau(s))-a(s)|\right\} \\
& +\operatorname{lE}\left\{1+\sum_{s=n_{0}}^{n-1}|h(s)| \prod_{u=s+1}^{n-1} H(u)\right\} \leq \alpha f(l) .
\end{aligned}
$$

Then there exists $\delta>0$ such that for any $\left.\psi:\left[m n_{0}\right), n_{0}\right] \cap \mathbf{Z} \rightarrow(-\delta, \delta)$, we have that $P: S_{\psi}^{l} \rightarrow S_{\psi}^{l}$ and $P$ is a contraction mapping with respect to the metric defined on $S_{\psi}^{l}$.

Proof. Since $f$ is odd and satisfies the Lipshitz condition on $[-l, l]$, $f(0)=0$ and $f$ is uniformly continuous on $[-l, l]$. So we can choose a $\delta$ that satisfies

$$
\delta\left(1+E+K \sum_{s=n_{0}-\tau\left(n_{0}\right)}^{n_{0}-1}|h(s)|\right) \leq(1-\alpha) l .
$$

Let $\psi \in D\left(n_{0}\right)$ such that $|\psi(n)| \leq \delta$ for $n \in\left[m\left(n_{0}\right), n_{0}\right] \cap \mathbf{Z}$. Note that (2.8) implies $\delta<l$ since $f(l) \leq l$ by condition (ii). Thus, $|\psi(n)| \leq l$ for $n \in\left[m\left(n_{0}\right), n_{0}\right] \cap \mathbf{Z}$. Now we show that for such a $\psi$ the mapping $P: S_{\psi}^{l} \rightarrow S_{\psi}^{l}$. Indeed, consider (2.7). For an arbitrary $\varphi \in S_{\psi}^{l}$, if follows from conditions $(i)$ and $(i i)$ that

$$
\begin{aligned}
& |(P \varphi)(n)| \leq\left\{(1+E)\|\psi\|+\sum_{s=n_{0}-\tau\left(n_{0}\right)}^{n_{0}-1}|h(s)||f(\psi(s))|\right\} \prod_{u=n_{0}}^{n-1} H(u) \\
& +\sum_{s=n_{0}}^{n-1}|h(s)| \prod_{u=s+1}^{n-1} H(u)|\varphi(s)-f(\varphi(s))| \\
& +\sum_{s=n-\tau(n)}^{n-1}|h(s)||f(\varphi(s))| \\
& +\sum_{s=n_{0}}^{n-1}|h(s)| \prod_{u=s+1}^{n-1} H(u) \sum_{v=s-\tau(s)}^{s-1}|h(v)||f(\varphi(v))| \\
& +\sum_{s=n_{0}}^{n-1} \prod_{u=s+1}^{n-1} H(u)|h(s-\tau(s))-a(s)||f(\varphi(s-\tau(s)))| \\
& +|g(n, \varphi(n-\tau(n)))|+\sum_{s=n_{0}}^{n-1}|h(s)| \prod_{u=s+1}^{n-1} H(u)|g(s, \varphi(s-\tau(s)))|
\end{aligned}
$$




$$
\begin{aligned}
& \leq \delta\left(1+E+K \sum_{s=n_{0}-\tau\left(n_{0}\right)}^{n_{0}-1}|h(s)|\right) \\
& +(l-f(l)) \sum_{s=n_{0}}^{n-1}|h(s)| \prod_{u=s+1}^{n-1} H(u)+f(l) \sum_{s=n-\tau(n)}^{n-1}|h(s)| \\
& +f(l) \sum_{s=n_{0}}^{n-1}|h(s)| \prod_{u=s+1}^{n-1} H(u) \sum_{v=s-\tau(s)}^{s-1}|h(v)| \\
& +f(l) \sum_{s=n_{0}}^{n-1} \prod_{u=s+1}^{n-1} H(u)|h(s-\tau(s))-a(s)| \\
& +l E\left\{1+\sum_{s=n_{0}}^{n-1}|h(s)| \prod_{u=s+1}^{n-1} H(u)\right\}
\end{aligned}
$$

for $n \in\left[n_{0}, \infty\right) \cap \mathbf{Z}$. By applying (iv) and (2.8), we see that

$$
\begin{aligned}
|(P \varphi)(n)| \leq \delta & \left(1+E+K \sum_{s=n_{0}-\tau\left(n_{0}\right)}^{n_{0}-1}|h(s)|\right)+(l-f(l)) \alpha+f(l) \alpha \\
& \leq(1-\alpha) l+(l-f(l)) \alpha+f(l) \alpha=l .
\end{aligned}
$$

Hence, $|(P \varphi)(n)| \leq \mathbf{Z}$ because $|(P \varphi)(n)|=|\psi(n)| \leq l$ for $n \in\left[m\left(n_{0}\right), n_{0}\right] \cap \mathbf{Z}$. Therefore, $P \varphi \in S_{\psi}^{l}$.

Suppose that $\rho>\max \{6,1 / K\}$. If we define a metric on $S_{\psi}^{l}$ as follows,

$$
\begin{aligned}
& |\varphi-\eta|_{\rho} \\
& :=\sup _{n \in\left[n_{0}, \infty\right) \cap \mathbf{Z}} \prod_{u=n_{0}}^{n-1} \frac{[1-|h(u)|][1-|h(s-\tau(u))-a(u)|][1-E|h(u)| / K]}{\rho K[1+|h(u)|][1+|h(u-\tau(u))-a(u)|][1+E|h(u)| / K]} \\
& \times|\varphi(n)-\eta(n)|,
\end{aligned}
$$

then $\left(S_{\psi}^{l},|\cdot|_{\rho}\right)$ is a complete metric space.

Next, we show that $P$ is a contraction mapping on $S_{\psi}^{l}$ with respect to the metric (2.9). For $\varphi, \eta \in S_{\psi}^{l}$, we have

$$
|(P \varphi)(n)-(P \eta)(n)|
$$




$$
\begin{aligned}
& \leq \sum_{s=n_{0}}^{n-1}|h(s)| \prod_{u=s+1}^{n-1} H(u)|\varphi(s)-f(\varphi(s))-\eta(s)+f(\eta(s))| \\
& +\sum_{s=n-\tau(n)}^{n-1}|h(s)||f(\varphi(s))-f(\eta(s))| \\
& +\sum_{s=n_{0}}^{n-1}|h(s)| \prod_{u=s+1}^{n-1} H(u) \sum_{v=s-\tau(s)}^{s-1}|h(v)||f(\varphi(v))-f(\eta(v))| \\
& +\sum_{s=n_{0}}^{n-1} \prod_{u=s+1}^{n-1} H(u)|h(s-\tau(s))-a(s)||f(\varphi(s-\tau(s)))-f(\eta(s-\tau(s)))| \\
& +|g(n, \varphi(n-\tau(n)))-g(n, \eta(n-\tau(n)))| \\
& +\sum_{s=n_{0}}^{n-1}|h(s)| \prod_{u=s+1}^{n-1} H(u)|g(s, \varphi(s-\tau(s)))-g(s, \eta(s-\tau(s)))| .
\end{aligned}
$$

Let $F(x)=x-f(x)$, then $F(x)$ satisfies a Lipschitz condition with constant $K>0$ on an interval $[-l, l]$. If we multiply both sides of $(2.10)$ by

$$
\prod_{u=n_{0}}^{n-1} \frac{[1-|h(u)|][1-|h(u-\tau(u))-a(u)|][1-E|h(u)| / K]}{\rho K[1+|h(u)|][1+|h(u-\tau(u))-a(u)|][1+E|h(u)| / K]},
$$

then the first term on the right-hand side of (2.10) becomes

$$
\begin{aligned}
& \prod_{u=n_{0}}^{n-1} \frac{[1-|h(u)|][1-|h(u-\tau(u))-a(u)|][1-E|h(u)| / K]}{\rho K[1+|h(u)|][1+|h(u-\tau(u))-a(u)|][1+E|h(u)| / K]} \\
& \times \sum_{s=n_{0}}^{n-1}|h(s)| \prod_{u=s+1}^{n-1} H(u)|F(\varphi(s))-F(\eta(s))| \\
& \leq K \sum_{s=n_{0}}^{n-1} \frac{|h(s)|[1-|h(s)|][1-|h(s-\tau(s))-a(s)|][1-E|h(s)| / K]}{\rho K[1+|h(s)|][1+|h(s-\tau(s))-a(s)|][1+E|h(s)| / K]} \\
& \times \prod_{u=n_{0}}^{s-1} \frac{[1-|h(u)|][1-|h(u-\tau(u))-a(u)|][1-E|h(u)| / K]}{\rho K[1+|h(u)|[1+|h(u-\tau(u))-a(u)|[1+E|h(u)| / K]}|\varphi(s)-\eta(s)| \\
& \times \prod_{u=s+1}^{n-1} \frac{H(u)[1-|h(u)|][1-|h(u-\tau(u))-a(u)|][1-E|h(u)| / K]}{\rho K[1+|h(u)|][1+|h(u-\tau(u))-a(u)|][1+E|h(u)| / K]} \\
& \leq K \frac{1}{\rho K}|\varphi-\eta|_{\rho} \sum_{s=n_{0}}^{n-1}|h(s)| \prod_{u=s+1}^{n-1}[1-|h(u)|] \\
& \leq \frac{1}{\rho}|\varphi-\eta|_{\rho} .
\end{aligned}
$$


Similarly, we have

$$
\begin{aligned}
& \prod_{u=n_{0}}^{n-1} \frac{[1-|h(u)|][1-|h(u-\tau(u))-a(u)|][1-E|h(u)| / K]}{\rho K[1+|h(u)|][1+|h(u-\tau(u))-a(u)|][1+E|h(u)| / K]} \\
& \times \sum_{s=n-\tau(n)}^{n-1}|h(s)||f(\varphi(s))-f(\eta(s))| \\
& \leq K \sum_{s=n_{0}}^{n-1} \frac{|h(s)|[1-|h(s)|][1-|h(s-\tau(s))-a(s)|][1-E|h(s)| / K]}{\rho K[1+|h(s)| \mid[1+|h(s-\tau(s))-a(s)| \mid[1+E|h(s)| / K]} \\
& \times \prod_{u=n_{0}}^{s-1} \frac{[1-|h(u)|][1-|h(u-\tau(u))-a(u)|][1-E|h(u)| / K]}{\rho K[1+|h(u)|][1+|h(u-\tau(u))-a(u)|[1+E|h(u)| / K]}|\varphi(s)-\eta(s)| \\
& \times \prod_{u=s+1}^{n-1} \frac{[1-|h(u)|][1-|h(u-\tau(u))-a(u)|][1-E|h(u)| / K]}{\rho K[1+|h(u)|][1+|h(u-\tau(u))-a(u)|][1+E|h(u)| / K]} \\
& \leq K \frac{1}{\rho K}|\varphi-\eta|_{\rho} \sum_{s=n_{0}}^{n-1}|h(s)| \prod_{u=s+1}^{n-1}[1-|h(u)|] \\
& \leq \frac{1}{\rho}|\varphi-\eta|_{\rho} \\
& \prod_{u=n_{0}}^{n-1} \frac{[1-|h(u)|][1-|h(u-\tau(u))-a(u)|][1-E|h(u)| / K]}{\rho K[1+|h(u)|][1+|h(u-\tau(u))-a(u)|][1+E|h(u)| / K]} \\
& \times \sum_{s=n_{0}}^{n-1}|h(s)| \prod_{u=s+1}^{n-1} H(u) \sum_{v=s-\tau(s)}^{s-1}|h(v)||f(\varphi(v))-f(\eta(v))| \\
& \leq K \sum_{s=n_{0}}^{n-1} \frac{|h(s)|[1-|h(s)|][1-|h(s-\tau(s))-a(s)|][1-E|h(s)| / K]}{\rho K[1+|h(s)| \mid[1+|h(s-\tau(s))-a(s)| \mid[1+E|h(s)| / K]} \\
& \times \prod_{u=s+1}^{n-1} \frac{H(u)[1-|h(u)|][1-|h(u-\tau(u))-a(u)|][1-E|h(u)| / K]}{\rho K[1+|h(u)|[1+|h(u-\tau(u))-a(u)|][1+E|h(u)| / K]} \\
& \times \sum_{v=s-\tau(s)}^{s-1}|h(v)| \prod_{u=n_{0}}^{v-1} \frac{[1-|h(v)|][1-|h(v-\tau(v))-a(v)|][1-E|h(v)| / K]}{\rho K[1+|h(v)|][1+|h(v-\tau(v))-a(v)|][1+E|h(v)| / K]}|\varphi(v)-\eta(v)| \\
& \times \prod_{u=v}^{s-1} \frac{[1-|h(v)|][1-|h(v-\tau(v))-a(v)|][1-E|h(v)| / K]}{\rho K[1+|h(v)|[1+|h(v-\tau(v))-a(v)| \mid[1+E|h(v)| / K]} \\
& \leq K \frac{1}{\rho K}|\varphi-\eta|_{\rho} \sum_{s=n_{0}}^{n-1}|h(s)| \prod_{u=s+1}^{n-1}[1-|h(u)|] \sum_{v=s-\tau(s)}^{s-1}|h(v)| \\
& \times \prod_{u=v}^{s-1}[1-|h(v)|] \\
& \leq \frac{1}{\rho}|\varphi-\eta|_{\rho},
\end{aligned}
$$




$$
\begin{aligned}
& \prod_{u=n_{0}}^{n-1} \frac{[1-|h(u)|][1-|h(u-\tau(u))-a(u)|][1-E|h(u)| / K]}{\rho K[1+|h(u)|][1+|h(u-\tau(u))-a(u)|][1+E|h(u)| / K]} \\
& \times \sum_{s=n_{0}}^{n-1} \prod_{u=s+1}^{n-1} H(u)|h(s-\tau(s))-a(s)| \mid \\
& \times f(\varphi(s-\tau(s)))-f(\eta(s-\tau(s))) \\
& \leq K \sum_{s=n_{0}}^{n-1} \frac{|h(s-\tau(s))-a(s)|[1-|h(s)|][1-|h(s-\tau(s))-a(s)|][1-E|h(s)| / K]}{\rho K[1+|h(s)|[1+|h(s-\tau(s))-a(s)|][1+E|h(s)| / K]} \\
& \times \prod_{u=n_{0}}^{s-1} \frac{[1-|h(u)|][1-|h(u-\tau(u))-a(u)|][1-E|h(u)| / K]}{\rho K[1+|h(u)|][1+|h(u-\tau(u))-a(u)|][1+E|h(u)| / K]}|\varphi(s)-\eta(s)| \\
& \times \prod_{u=s+1}^{n-1} \frac{H(u)[1-|h(u)|][1-|h(u-\tau(u))-a(u)|][1-E|h(u)| / K]}{\rho K[1+|h(u)|][1+|h(u-\tau(u))-a(u)|][1+E|h(u)| / K]} \\
& \leq K \frac{1}{\rho K}|\varphi-\eta|_{\rho} \sum_{s=n_{0}}^{n-1}|h(s-\tau(s))-a(s)| \\
& \times \prod_{u=s+1}^{n-1}[1-|h(u-\tau(u))-a(u)|] \\
& \leq \frac{1}{\rho}|\varphi-\eta|_{\rho}, \\
& \prod_{u=n_{0}}^{n-1} \frac{[1-|h(u)|][1-|h(u-\tau(u))-a(u)|][1-E|h(u)| / K]}{\rho K[1+|h(u)|][1+|h(u-\tau(u))-a(u)|][1+E|h(u)| / K]} \\
& \times|g(n, \varphi(n-\tau(n)))-g(n, \eta(n-\tau(n)))| \\
& \leq E|\varphi-\eta|_{\rho} \leq \frac{1}{\rho}|\varphi-\eta|_{\rho},
\end{aligned}
$$

and

$$
\begin{aligned}
& \prod_{u=n_{0}}^{n-1} \frac{[1-|h(u)|][1-|h(u-\tau(u))-a(u)|][1-E|h(u)| / K]}{\rho K[1+|h(u)|][1+|h(u-\tau(u))-a(u)|][1+E|h(u)| / K]} \\
& \times \sum_{s=n_{0}}^{n-1}|h(s)| \prod_{u=s+1}^{n-1} H(u)|g(s, \varphi(s-\tau(s)))-g(s, \eta(s-\tau(s)))| \\
& \leq E \sum_{s=n_{0}}^{n-1} \frac{|h(s)|[1-|h(s)|][1-|h(s-\tau(s))-a(s)|][1-E|h(s)| / K]}{\rho K[1+|h(s)|[1+|h(s-\tau(s))-a(s)|[1+E|h(s)| / K]} \\
& \times \prod_{u=n_{0}}^{s-1} \frac{[1-|h(u)|][1-|h(u-\tau(u))-a(u)|][1-E|h(u)| / K]}{\rho K[1+|h(u)|[1+|h(u-\tau(u))-a(u)|][1+E|h(u)| / K]}|\varphi(s)-\eta(s)| \\
& \times \prod_{u=s+1}^{n-1} \frac{H(u)[1-|h(u)|][1-|h(u-\tau(u))-a(u)|][1-E|h(u)| / K]}{\rho K[1+|h(u)|][1+|h(u-\tau(u))-a(u)|[1+E|h(u)| / K]}
\end{aligned}
$$


$\leq K \frac{1}{\rho K}|\varphi-\eta|_{\rho} \sum_{s=n_{0}}^{n-1} E|h(s)| / K \prod_{u=s+1}^{n-1}[1-E|h(u)| / K]$

$\leq \frac{1}{\rho}|\varphi-\eta|_{\rho}$

Hence, $|P \varphi-P \eta|_{\rho} \leq \frac{6}{\rho}|\varphi-\eta|_{\rho}$, since $\rho>6$, we have that $P$ is a contraction mapping on $S_{\psi}^{l}$.

Theorem 2.4. Assume that the hypotheses of Theorem 2.3 hold. Then the zero solution of (1.1) is stable.

Proof. Let $P$ be defined as in Theorem 2.3. By the contraction mapping principle ([26], p. 2), $P$ has a unique fixed point in $S_{\psi}^{l}$, which is a solution of $(1.1)$ with $x=\psi$ on $\left[m\left(n_{0}\right), n_{0}\right] \cap \mathbf{Z}$.

To prove stability at $n=n_{0}$, let $\epsilon>0$ be given, then we choose $m>0$ so that $m<\min \{l, \epsilon\}$. By considering $S_{\psi}^{m}$, we obtain there is a $\delta>0$ such that $\|\psi\|<\delta$ implies that the unique solution of (1.1) with $x=\psi$ on $\left[m\left(n_{0}\right), n_{0}\right] \cap \mathbf{Z}$ satisfies $|x(n)| \leq m<\epsilon$ for all $n \in\left[m\left(n_{0}\right), \infty\right) \cap \mathbf{Z}$. This proves that the zero solution of (1.1) is stable.

Definition 2.5. The zero solution of (1.1) is asymptotically stable if it is Lyapunov stable and if for any integer $n_{0} \geq 0$ there exists a $\delta>0$ such that $|\psi(n)| \leq \delta$ for $n \in\left[m\left(n_{0}\right), n_{0}\right] \cap \mathbf{Z}$ implies $x\left(n, n_{0}, \psi\right) \rightarrow 0$ as $n \rightarrow \infty$.

Theorem 2.6. Assume that the hypotheses of Theorem 2.3 hold. Also assume that

$$
\prod_{u=n_{0}}^{n-1} H(u) \rightarrow 0 \text { as } n \rightarrow \infty,
$$

where $H(u)=1-h(u)$. Then the zero solution of (1.1) is asymptotically stable. 
Proof. From Theorem 2.4, the zero solution of (1.1) is stable. For a given $\epsilon>0$ let $\psi \in D\left(n_{0}\right)$ such that $|\psi(n)| \leq \delta$ for $n \in\left[m\left(n_{0}\right), n_{0}\right] \cap \mathbf{Z}$ where $\delta>0$ and define

$$
\begin{gathered}
S_{\psi}^{\epsilon}=\left\{\varphi \in C, \varphi(n)=\psi(n) \text { for } n \in\left[m\left(n_{0}\right), n_{0}\right] \cap \mathbf{Z},\|\varphi\| \leq \epsilon\right. \text { and } \\
\varphi(n) \rightarrow 0 \text { as } n \rightarrow \infty .
\end{gathered}
$$

Then $S_{\psi}^{\epsilon}$ is a complete metric space with respect to the metric (2.9). Define $P: S_{\psi}^{\epsilon} \rightarrow S_{\psi}^{\epsilon}$ by (2.9). From the proof of Theorem 2.3, the mapping $P$ is a contraction and for every $\varphi \in S_{\psi}^{\epsilon},\|P \varphi\| \leq \epsilon$.

We next show that $(P \varphi)(n) \rightarrow 0$ as $n \rightarrow \infty$. There are seven terms on the right hand side in (2.7). Denote them, respectively, by $I_{k}, k=1,2, \ldots, 7$. It is obvious that the first term $I_{1}$ tends to zero as $t \rightarrow \infty$, by condition (2.11). Therefore, the second term $I_{2}$ in (2.7) satisfies

$$
\begin{aligned}
&\left|I_{2}\right|=\left|\sum_{s=n_{0}}^{n-1} h(s) \prod_{u=s+1}^{n-1} H(u)[\varphi(s)-f(\varphi(s))]\right| \\
& \leq K \sum_{s=n_{0}}^{n-1}|h(s)| \prod_{u=s+1}^{n-1} H(u)|\varphi(s)| \\
& \leq \epsilon K \alpha<\epsilon K .
\end{aligned}
$$

Thus, $I_{2} \rightarrow 0$ as $n \rightarrow \infty$. Also, due to the conditions (1.2) and (1.3) and the facts that $\varphi(n) \rightarrow 0$ and $n-\tau(n) \rightarrow \infty$ as $n \rightarrow \infty$, the terms $I_{3}$ and $I_{6}$ tend to zero, as $n \rightarrow \infty$.

Now, for a given $\epsilon_{1} \in(0, \epsilon)$, there exists a $N_{1}>n_{0}$ such that $s \geq N_{1}$ implies $\left|\varphi\left(s-\tau_{j}(s)\right)\right|<\epsilon_{1}$. Thus, for $n \geq N_{1}$, the term $I_{4}$ in (2.7) satisfies

$$
\begin{aligned}
& \left|I_{4}\right|=\left|\sum_{s=n_{0}}^{n-1} h(s) \prod_{u=s+1}^{n-1} H(u) \sum_{v=s-\tau(s)}^{s-1} h(v) f(\varphi(v))\right| \\
& \leq \sum_{s=n_{0}}^{N_{1}-1}|h(s)| \prod_{u=s+1}^{n-1} H(u) \sum_{v=s-\tau(s)}^{s-1}|h(v)||f(\varphi(v))| \\
& +\sum_{s=N_{1}}^{n-1}|h(s)| \prod_{u=s+1}^{n-1} H(u) \sum_{v=s-\tau(s)}^{s-1}|h(v)||f(\varphi(v))| \\
& \leq \sup _{\sigma \geq m\left(n_{0}\right)}|\varphi(\sigma)| K \sum_{s=n_{0}}^{N_{1}-1}|h(s)| \prod_{u=s+1}^{n-1} H(u) \sum_{v=s-\tau(s)}^{s-1}|h(v)| \\
& +\epsilon_{1} K \sum_{s=N_{1}}^{n-1}|h(s)| \prod_{u=s+1}^{n-1} H(u) \sum_{v=s-\tau(s)}^{s-1}|h(v)| .
\end{aligned}
$$

By (2.11), we can find $N_{2}>N_{1}$ such that $n \geq N_{2}$ implies 


$$
\begin{aligned}
& \sup _{\sigma \geq m\left(n_{0}\right)}|\varphi(\sigma)| K \sum_{s=n_{0}}^{N_{1}-1}|h(s)| \prod_{u=s+1}^{n-1} H(u) \sum_{v=s-\tau(s)}^{s-1}|h(v)| \\
= & \sup _{\sigma \geq m\left(n_{0}\right)}|\varphi(\sigma)| K \prod_{u=N_{2}}^{n-1} H(u) \sum_{s=n_{0}}^{N_{1}-1}|h(s)| \prod_{u=s+1}^{N_{2}-1} H(u) \sum_{v=s-\tau(s)}^{s-1}|h(v)| \\
< & \epsilon_{1} K .
\end{aligned}
$$

Now, apply condition (iv) to have $\left|I_{4}\right|<\epsilon_{1} K+\epsilon_{1} K \alpha<2 \epsilon_{1} K$. Thus, $I_{4} \rightarrow 0$ as $n \rightarrow \infty$. Similarly, by using (2.11), then, if $n \geq N_{2}$ then terms $I_{5}$ and in $I_{7}(2.7)$ satisfy

$$
\begin{aligned}
& \left|I_{5}\right|=\left|\sum_{s=n_{0}}^{n-1} \prod_{u=s+1}^{n-1} H(u)\{h(s-\tau(s))-a(s)\} f(\varphi(s-\tau(s)))\right| \\
& \leq \sup _{\sigma \geq m\left(n_{0}\right)}|\varphi(\sigma)| K \prod_{u=N_{2}}^{n-1} H(u) \sum_{s=n_{0}}^{N_{1}-1} \prod_{u=s+1}^{N_{2}-1} H(u)|h(s-\tau(s))-a(s)| \\
& +\epsilon_{1} K \sum_{s=N_{1}}^{n-1} \prod_{u=s+1}^{n-1} H(u)|h(s-\tau(s))-a(s)| \\
& \leq \epsilon_{1} K+\epsilon_{1} K \alpha<2 \epsilon_{1} K
\end{aligned}
$$

and

$$
\begin{aligned}
& \left|I_{7}\right|=\left|\sum_{s=n_{0}}^{n-1} h(s) \prod_{u=s+1}^{n-1} H(u) g(s, \varphi(s-\tau(s)))\right| \\
& \leq \sup _{\sigma \geq m\left(n_{0}\right)}|\varphi(\sigma)| E \prod_{u=N_{2}}^{n-1} H(u) \sum_{s=n_{0}}^{N_{1}-1}|h(s)| \\
& \times \prod_{u=s+1}^{N_{2}-1} H(u)+\epsilon_{1} E \sum_{s=N_{1}}^{n-1}|h(s)| \prod_{u=s+1}^{n-1} H(u) \\
& \leq \epsilon_{1}+\epsilon_{1} \alpha \frac{f(l)}{l}<\left(1+\frac{f(l)}{l}\right) \epsilon_{1} .
\end{aligned}
$$

Thus, $I_{5}, I_{7} \rightarrow 0$ as $n \rightarrow \infty$. In conclusion $(P \varphi)(n) \rightarrow 0$ as $n \rightarrow \infty$, as required. Hence $P$ maps $S_{\psi}^{\epsilon}$ into $S_{\psi}^{\epsilon}$.

By the contraction mapping principle, $P$ has a unique fixed point $x \in S_{\psi}^{\epsilon}$ which solves (1.1). Therefore, the zero solution of (1.1) is asymptotically stable.

Letting $g(n, x)=0$, we have

Corollary 2.7. Let $h:\left[m\left(n_{0}\right), \infty\right) \cap \mathbf{Z} \rightarrow \mathbf{R}$ be an arbitrary sequence. Suppose that the following conditions are satisfied, 
(i) the function $f$ is odd, increasing on $[0, l]$,

(ii) $f(x)$ and $x-f(x)$ satisfy a Lipschitz condition with constant $K$ on an interval $[-l, l]$, and $x-f(x)$ is nondecreasing on $[0, l]$,

(iii) $|h(n)|<1$ for $n \in\left[m\left(n_{0}\right), \infty\right) \cap \mathbf{Z}$ and $|h(n-\tau(n))-a(n)|<1$ for $n \in\left[n_{0}, \infty\right) \cap \mathbf{Z}$,

(iv) there exist constants $\alpha \in(0,1)$ for all $n \in\left[n_{0}, \infty\right) \cap \mathbf{Z}$ such that

$$
\begin{aligned}
& \sum_{s=n_{0}}^{n-1}|h(s)| \prod_{u=s+1}^{n-1} H(u)+\sum_{s=n-\tau(n)}^{n-1}|h(s)| \\
& +\sum_{s=n_{0}}^{n-1}|h(s)| \prod_{u=s+1}^{n-1} H(u) \sum_{v=s-\tau(s)}^{s-1}|h(v)| \\
& +\sum_{s=n_{0}}^{n-1} \prod_{u=s+1}^{n-1} H(u)|h(s-\tau(s))-a(s)| \\
& \leq \alpha .
\end{aligned}
$$

Then the zero solution of (1.7) is asymptotically stable if

$$
\prod_{u=n_{0}}^{n-1} H(u) \rightarrow 0 \text { as } n \rightarrow \infty .
$$

Remark 2.8. When $\tau(s)=r$ and $h(s)=a(s+r)$, Corollary 2.7 improves Theorem $C$. When $h(s)=a(g(s))$, where $g(s)$ is the inverse function of $s-\tau(s)$, Corollary 2.7 reduces to Theorem D. Thus Theorem 2.6 generalizes and improves Theorems $C$ and $D$.

For the special case $g(n, x)=0$ and $f(x)=x$, we can get

Corollary 2.9. Suppose that $H(n) \neq 0$ for all $n \in\left[n_{0}, \infty\right) \cap \mathbf{Z}$ and there exists a constant $\alpha \in(0,1)$ such that for $n \in\left[n_{0}, \infty\right) \cap \mathbf{Z}$

$$
\begin{aligned}
& \sum_{s=n-\tau(n)}^{n-1}|h(s)|+\sum_{s=n_{0}}^{n-1}|h(s)| \prod_{u=s+1}^{n-1}|H(u)| \sum_{v=s-\tau(s)}^{s-1}|h(v)| \\
& +\sum_{s=n_{0}}^{n-1} \prod_{u=s+1}^{n-1}|H(u)||h(s-\tau(s))-a(s)| \\
& \leq \alpha .
\end{aligned}
$$

Then the zero solution of (1.4) is asymptotically stable if

$$
\prod_{u=n_{0}}^{n-1} H(u) \rightarrow 0 \text { as } n \rightarrow \infty .
$$


Remark 2.10. When $\tau(s)=r$ and $h(s)=a(s+r)$, Corollary 2.9 reduces to Theorem $A$. When $h(s)=a(g(s))$, where $g(s)$ is the inverse function of $s-\tau(s)$, Corollary 2.9 reduces to Theorem B. Thus Theorem 2.6 improves Theorems $A$ and $B$.

\section{References}

[1] A. Ardjouni and A. Djoudi, Fixed points and stability in linear neutral differential equations with variable delays, Nonlinear Analysis 74, pp. 2062-2070, (2011).

[2] A. Ardjouni and A. Djoudi, Stability in nonlinear neutral integrodifferential equations with variable delay using fixed point theory, J. Appl. Math. Comput. 44 : pp. 317-336, (2014).

[3] A. Ardjouni and A. Djoudi, Stability in nonlinear neutral difference equations, Afr. Mat. 26 : pp. 559-574, (2015).

[4] L. Berezansky and E. Braverman, On exponential dichotomy, BohlPerron type theorems and stability of difference equations, J. Math. Anal. Appl. 304, pp. 511-530, (2005).

[5] L. Berezansky, E. Braverman and E. Liz, Sufficient conditions for the global stability of nonautonomous higher order difference equations, J. Difference Equ. Appl. 11, No. 9, pp. 785-798, (2005).

[6] T. A. Burton, Stability by Fixed Point Theory for Functional Differential Equations, Dover, New York, (2006).

[7] T. A. Burton and T. Furumochi, Fixed points and problems in stability theory, Dynam. Systems Appl. 10, pp. 89-116, (2001).

[8] G. E. Chatzarakis, G.N. Miliaras, Asymptotic behavior in neutral difference equations with variable coefficients and more than one delay arguments. J. Math. Comput. Sci. 1, No. 1, pp. 32-52, (2011).

[9] G. Chen, A fixed point approach towards stability of delay differential equations with applications to neural networks, Ph. D. thesis, Leiden University, (2013). 
[10] S. Elaydi, An Introduction to Difference Equations, Springer, New York, (1999).

[11] S. Elaydi, Periodicity and stability of linear Volterra difference systems, J. Math. Anal. Appl. 181, pp. 483-492, (1994).

[12] S. Elaydi and S. Murakami, Uniform asymptotic stability in linear Volterra difference equations, J. Difference Equ. Appl. 3, pp. 203-218, (1998).

[13] P. Eloe, M. Islam and Y. N. Raffoul, Uniform asymptotic stability in nonlinear Volterra discrete systems, Special Issue on Advances in Difference Equations IV, Computers Math. Appl. 45, pp. 1033-1039, (2003).

[14] I. Gyori and F. Hartung, Stability in delay perturbed differential and difference equations, Fields Inst. Commun. 29, pp. 181-194, (2001).

[15] M. Islam and Y. N. Raffoul, Exponential stability in nonlinear difference equations, J. Difference Equ. Appl. 9, pp. 819-825, (2003).

[16] M. Islam and E. Yankson, Boundedness and stability in nonlinear delay difference equations employing fixed point theory, Electronic Journal of Qualitative Theory of Differential Equations, No. 26, pp. 1-18, (2005).

[17] C. Jin and J. Luo, Stability by fixed point theory for nonlinear delay difference equations, Georgian Mathematical Journal, Volume 16, Number 4, pp. 683-691, (2009).

[18] W. G. Kelly and A. C. Peterson, Difference Equations : An Introduction with Applications, Academic Press, (2001).

[19] E. Liz, Stability of non-autonomous difference equations: simple ideas leading to useful results. J. Difference Equ. Appl. 17, No. 2, pp. 203220, (2011).

[20] E. Liz, On explicit conditions for the asymptotic stability of linear higher order difference equations, J. Math. Anal. Appl. 303, No. 2, pp. 492-498, (2005).

[21] V. V. Malygina and A. Y. Kulikov, On precision of constants in some theorems on stability of difference equations, Func. Differ. Equ. 15, No. 3-4, pp. 239-249, (2008). 
[22] M. Pituk, A criterion for the exponential stability of linear difference equations, Appl. Math. Lett. 17, pp. 779-783, (2004).

[23] Y. N. Raffoul, Stability and periodicity in discrete delay equations. J. Math. Anal. Appl. 324, No. 2, pp. 1356-1362, (2006).

[24] Y. N. Raffoul, Periodicity in general delay nonlinear difference equations using fixed point theory. J. Difference Equ. Appl. 10, No. 13-15, pp. 1229-1242, (2004).

[25] Y. N. Raffoul, General theorems for stability and boundedness for nonlinear functional discrete systems, J. math. Anal. Appl. 279, pp. 639-650, (2003).

[26] D. R. Smart, Fixed point theorems, Cambridge Tracts in Mathematics, No. 66. Cambridge University Press, London-New York, (1974).

[27] E. Yankson, Stability in discrete equations with variable delays, Electronic Journal of Qualitative Theory of Differential Equations, No. 8, pp. 1-7, (2009).

[28] E. Yankson, Stability of Volterra difference delay equations, Electronic Journal of Qualitative Theory of Differential Equations, No. 20, pp. 1-14, (2006).

[29] B. Zhang, Fixed points and stability in differential equations with variable delays, Nonlinear Analysis 63, e233-e242, (2005).

[30] B. G. Zhang, C. J. Tian and P. J. Y. Wong, Global attractivity of difference equations with variable delay, Dynam. Contin. Discrete Impuls. Systems 6, No. 3, pp. 307-317, (1999).

\author{
Abdelouaheb Ardjouni \\ Faculty of Sciences and Technology, \\ Department of Mathematics and Informatics, \\ University Souk Ahras, \\ P. O. Box 1553, \\ Souk Ahras, 41000, \\ Algeria \\ e-mail: abd_ardjouni@yahoo.fr \\ and
}




\begin{abstract}
Ahcene Djoudi
Applied Mathematics Lab,

Faculty of Sciences,

Department of Mathematics,

University Annaba,

P. O. Box 12, Annaba 23000,

Algeria

e-mail: adjoudi@yahoo.com
\end{abstract}

